\title{
Considerations for Hospital Approval of Human Participant Research
}

Cheryl M. Chanaud, PhD, CCRP

Memorial Hermann Hospital System, Houston, Texas, and University of Texas Health Science Center, Houston, Texas.

Disclosure: Nothing to report.

BACKGROUND Hospitals often accept as sufficient the federal requirement that human participant research studies have Institutional Review Board (IRB) review and approval, but IRBs usually do not consider many practical matters that arise in the implementation and operation of an interventional clinical trial in the complex environment of the modern acute care hospital.

METHODS A large hospital system in Texas implemented a policy requiring that any activity associated with human participant research receive prior hospital system review and approval. This hospital system review and approval process, which occurs in parallel with IRB review, focuses separately on patient safety and operational, financial, and special risk issues.

RESULTS A centralized research institute proactively gathers information needed to evaluate a proposed research study and forwards this information to unit directors and other key personnel in the affected hospitals for their review. When all reviewers have signed off on the study and the institute is satisfied that all pertinent issues have been resolved, it authorizes the principal investigator to begin the study in the designated hospitals.

CONCLUSIONS This review and approval process reduces risk, benefiting patients, hospital personnel who support research studies, research teams, and hospitals as institutions. Journal of Hospital Medicine 2010;5:E17-E24. @ 2010 Society of Hospital Medicine.

Hospitals have important legal and ethical responsibilities for human participant research conducted within their facilities, such as ensuring that research complies with federal regulations and presents minimal risks to patients. Many hospitals accept as sufficient the federal requirement that human participant research studies have Institutional Review Board (IRB) review and approval. IRBs must review proposed research according to numerous criteria, such as scientific soundness, alignment with accepted ethics principles and weighing of benefit vs. risk to study participants. ${ }^{1,2}$ The legally required aspects of IRB review do not, however, include considering practical matters in implementing and operating an interventional clinical trial in the complex environment of the modern acute care hospital.

Our hospital system established a broad policy requiring internal review and formal approval of any human participant research conducted within any of its hospitals, including studies that enroll hospital patients or hospital employees, utilize hospital medical records, or request hospitalprovided services for research tests or procedures. The purpose of this paper is to describe this formal hospital system review and approval process, the reasons for implementing it, and the types of issues considered prior to our hospital system granting a principal investigator permission to conduct a study.

\section{Background}

Surprisingly little healthcare or medical literature exists regarding hospital responsibilities toward human subject research conducted on its premises. Much of the literature focuses on ethics issues, the nature of informed consent, and study design. As critical as these discussions are, they seldom address the numerous complex operational issues and challenges that implementation of a clinical trial can create in a hospital setting.

Flanders et al. ${ }^{3}$ make the case for hospitalists and specialists to work together to support research that includes inpatients as study participants. Moore and Goldberg ${ }^{4}$ discuss the ingredients of successfully developing a research program in a community hospital and mention the need to involve all affected hospital departments in the initial hospital review of a study, evaluating study impact on hospital workflow, and establishing processes related to budgeting and billing. Jamerson ${ }^{5}$ also makes the case for hospital departmental review and involvement, assessment of the ability to integrate study activities into the hospital structure, assessment of the resources needed to support the research, determination of whether the hospital will contribute financially to the research, and explicit decision making regarding the assumption of institutional risk.

Despite the recognition that US patients increasingly live with multiple chronic conditions ${ }^{6}$ and that clinical trial protocols have become more procedure and resource intensive and costly, ${ }^{7-9}$ there has not been a corollary recognition of the increasing need for hospitals to understand and manage research activities occurring within their facilities.

Our organization is a hospital system with 9 acute care hospitals, including an academic teaching hospital 
(affiliated with a university medical school) with a Level 1 Trauma Center, 1 specialty rehabilitation hospital, numerous specialized clinics, and a LifeFlight Program with a 6-helicopter fleet (Geisinger, Danville, PA). This system of hospitals serves the fourth largest metropolitan community in the US (Houston and Harris County in southeastern TX), with a population of nearly four million and a geographic spread of 1778 square miles. ${ }^{10,11}$ The hospital system has approximately 140,700 inpatient admissions per year and 586,000 outpatient visits.

Eight years ago, our hospital system adopted a corporate policy requiring that any activity associated with human participant research receive prior hospital system review and approval. Our organization considers this review process vital to: (1) maintaining our commitment to our Federalwide Assurance with the Office for Human Research Protections, (2) abiding by the Joint Commission requirements related to research, ${ }^{12}$ (3) protecting the safety and confidentiality of patients and employees who are potential or actual research participants, (4) protecting the confidentiality of participants' medical information, (5) assuring that legal fundamentals and good clinical practice $(\mathrm{GCH})^{13}$ are a part of study plans, (6) assuring that studies are operationally feasible, and (7) evaluating and minimizing risks to patients and risks to the organization.

\section{Review and Approval Process \\ Overview}

An investigator triggers a formal hospital system review by submitting study documents to 1 of the IRBs listed on the system's Federalwide Assurance through the electronic IRB system and completing the required hospital system's Research Application Form. The hospital system review occurs in parallel with the IRB review, not duplicating it but rather focusing separately on patient safety, operational and financial issues, and hospital risk issues.

Our Clinical Innovation and Research Institute manages the hospital system review. Upon electronic notification of a new study submission, an Institute Clinical Research Associate examines all study-related documents, including the completed Research Application Form and other submitted documents, such as the study protocol, the investigational product's Investigator's Brochure, consent forms, Food and Drug Administration (FDA) letters, survey questions, and diary and other data collection forms. The Associate may spend considerable time communicating with the investigator's research team, collecting missing information and building a complete study file, including identifying the affected hospitals and hospital departments. The Institute then provides study documents to the individuals responsible for hospital-level research review, and each affected hospital conducts its own internal review and approval process.

The hospital-level review process varies depending on the hospitals involved. The academic teaching hospital has the most detailed review process, due to the complexities and risks associated with the full spectrum of human participant research which occurs there (Hospital A in Figure 1). If a study affects this hospital, the Institute provides study documents to each affected Department Manager, the affected Service Line Chief, the Chief Medical Officer or Medical Director of each Intensive Care Unit within which the study will recruit and enroll patients, and the Infection Control Officer and Radiation Safety Officer, as appropriate.

The specialty rehabilitation hospital has a long-standing national reputation for its research programs; its Director of Research knows each investigator, reviews each study, and provides that hospital's administrative review (Hospital B). Seven of the system's community hospitals have either a Chief Executive Officer, Chief Nursing Officer, or Chief Financial Officer serve as the hospital's executive administrator responsible for research review, and this person distributes the study documents to the Chief Medical Officer, if deemed necessary, and to each affected department (Hospitals C-I). One of the smaller community hospitals participates in relatively few studies; the Chief Nursing Officer reviews and provides hospital-level approval (Hospital J). For retrospective studies requesting a clinical data set, the Institute provides the study documents to the Director of the Information Systems Department. All studies accessing patient data are provided to the system Privacy Officer for review and approval.

Studies may involve 1 or more hospitals; some have involved as many as 7 at once.

All reviewers also receive a standardized Research Study Evaluation Form for their written comments and recommendations (Approve, Disapprove, or Defer) regarding whether the hospital system should approve the study.

If a study requests hospital-provided research services, the Institute's Research Financial Coordinator develops a research budget listing the hospital charges that the researcher will incur for these tests and procedures.

Once reviewers return completed Evaluation Forms, the Institute Clinical Research Associate makes an initial determination whether the review process has satisfactorily answered all questions and resolved all outstanding issues. The Manager of Clinical Research Operations then examines the study file to ensure a satisfactory review. Finally, the system Executive Director for Clinical Research provides a Letter of Approval to the Principal Investigator, which serves as the agreement of terms for conducting the study within the hospital system. The letter contains standard stipulations, such as requiring the Principal Investigator to abide by federal law and International Conference on Harmonization (ICH) GCPs and the budget for the hospital-provided research tests and procedures. Additionally, it includes any stipulations unique to the study-for example, that the Principal Investigator will provide training to hospital personnel who will be operating nonhospital equipment. The Institute provides affected hospital departments with copies of the approval letter. Upon signing and returning the letter, the Principal Investigator may begin the study in the designated hospitals. 


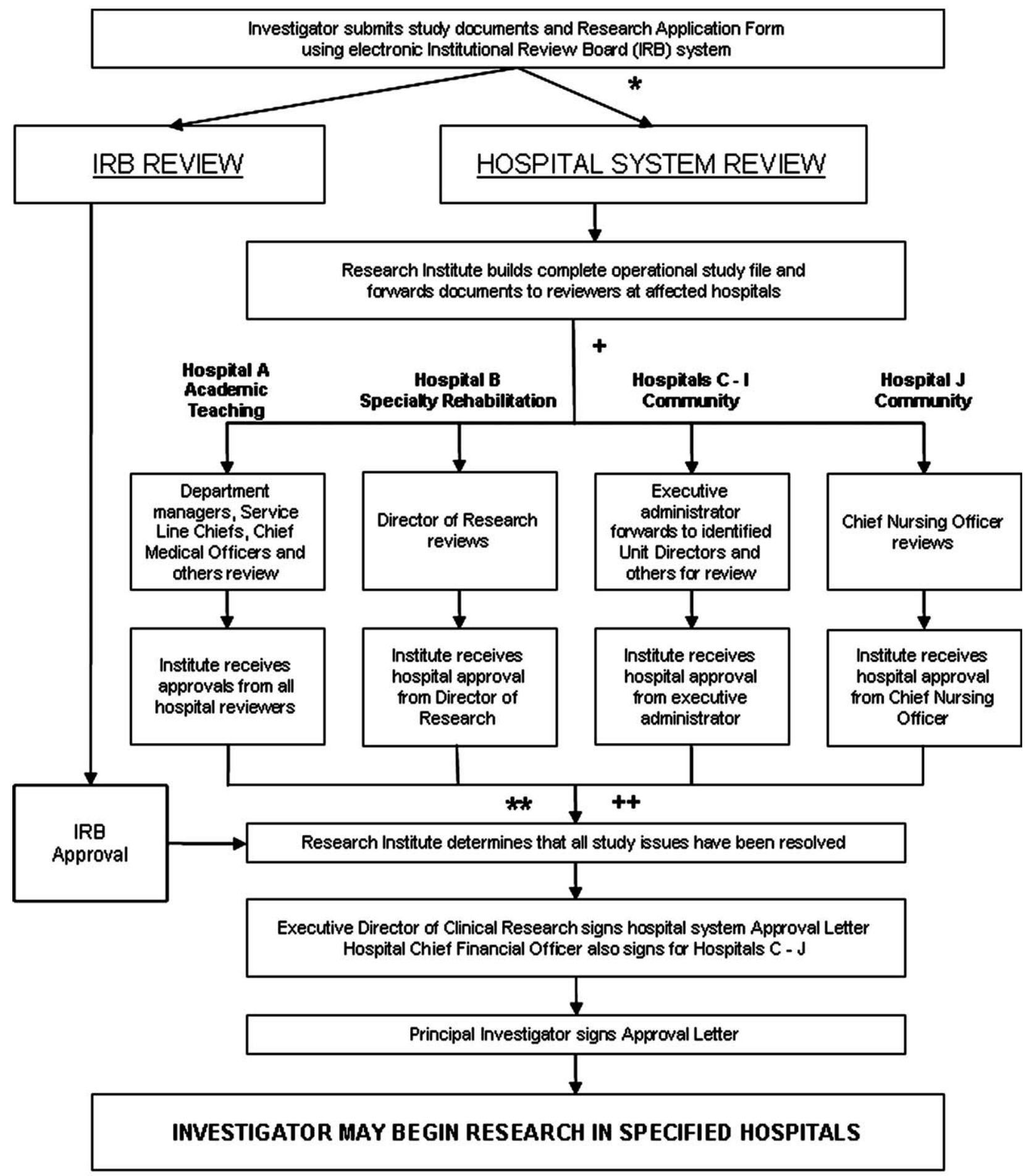

* Upon request, Institute prepares preliminary budget of researchrelated hospital services
+ Institute also sends studies accessing patient data to hospital system

Privacy Officer for review and approval
${ }^{* *}$ Institute sends studies requesting patient or hospital financial data to hospital Financial Officer for review and approval
++ Institute also sends studies with unusual risk issues to system and hospital Risk Officers for review and approval

FIGURE 1. Hospital system research review and approval process. 
Some details about the hospital system review are discussed in sections below.

\section{Patient Safety and Human Participant Protections Participant Recruitment Plans}

Sometimes IRB submission documents do not adequately describe how researchers will identify potential study participants and approach them for consent. Key concerns which we address include how researchers will identify potential participants in a Health Insurance Portability and Accountability Act (HIPAA)-compliant manner, the level and type of illnesses of the patients whom the investigator intends to recruit, whether the researcher must obtain the admitting or attending physician's permission, the qualifications of the person making the initial patient contact, and how and when that person will make contact, with special attention required if subjects may include very ill patients in an Intensive Care Unit (ICU).

\section{In-service for Unit Personnel and Pharmacists}

For most interventional studies, we require that the principal investigator's research team provide a plan for study education in-service for nurses, technicians, respiratory therapists, and pharmacists who may be involved in the care of a patient enrolled in the study. This is usually an inperson presentation done at a regular unit meeting, with an additional investigational drug-specific in-service provided and available on the hospital system intranet for the pharmacists. We remind researchers that the plan must also include an in-service for night shift personnel, who are often otherwise overlooked.

\section{Personnel Administering the Investigational Product}

Sometimes submitted protocols do not state who will administer the investigational product — the physician investigator, other members of the research team or unit nurses. If the investigator expects unit nurses to administer the product, the hospital needs to determine whether the nurses' experience and training qualifies them to administer it, assess for adverse events, and provide care for patients with these events. If the nurses are not qualified, then the hospital needs to decide whether the nurses should receive training or a member of the research team should administer the product. Some research studies involve investigational agents with novel administration techniques or risks of immediate severe adverse events, requiring the presence of a physician knowledgeable about the investigational product.

\section{Care of Study Participants With Adverse Events}

Studies with unusual investigational agents can also raise a Unit Director's or ICU Medical Director's concern as to whether bedside nurses can appropriately and adequately discern and respond to potential adverse events. If the investigational agent might result in an event not normally anticipated in patients in that particular unit, the hospital may need to consider additional preparation or staffing.

\section{Consent Documentation}

Federal law and ICH GCPs require that principal investigators have signed consent forms available in the research records, which may be off of the hospital premises in physician clinics or office areas. Our hospital system requires a copy of the consent form in the patient's medical chart if the research team conducted the consent process within the hospital or if the study participant will be an inpatient for a procedure included as part of the study protocol, whether the patient was recruited while an inpatient or prior to inpatient admission. This is important for meeting the Joint Commission's standards related to research. We established an internal monitoring program to verify that researchers were providing copies of consent, assent and parental permission forms to the Health Information Management Office for placement in medical charts.

\section{IRB-related Issues}

Occasionally a hospital system review identifies an IRBrelated concern, such as a known possible adverse event missing from the consent form, unexplained medical terms in the consent form, exclusion criteria not mentioning pregnancy or a consent form not covering a pregnancy test even though the protocol text mentions these, or missing Investigational New Drug Application (IND) or Investigational Device Exemption (IDE) information. Institute staff route such concerns to the IRB for follow-up with the researcher as necessary. If a hospital system review identifies an ethical concern, the Institute consults with the IRB Chair or ethicist member. Usually the Chair will assess the concern, raise options for addressing it, and recommend a course of action.

\section{Operational Issues}

\section{Feasibility and Implementation}

We urge researchers to meet with Institute staff to discuss implementation of a protocol as a real-world, operationalized study and also encourage them to meet with managers of the primary units where the study will take place. Researchers, however, may develop and submit industrysponsored clinical trials or investigator-initiated studies without such prior discussions and may not have adequately considered operational feasibility.

Given the increasing complexity of investigational agents, study designs, study procedures, and patient safety monitoring, hospital reviewers need to consider exactly who will perform study procedures and processes and how those people will do so. If a blood or tissue sample needs to be spun, packaged, and mailed on dry ice within a limited timeframe, for example, who will do this and will the supplies and equipment be available as needed? A study protocol can lead to a change in normal unit processes. 
Operating suite managers, for instance, may need to adjust schedules or work with research teams if research activities may prolong a procedure beyond average timeframes.

Other potential impacts abound. Research teams sometimes assume, without checking with hospital managers, that hospital staff, usually nurses or respiratory therapists, will perform research procedures of the kind they usually perform as standard clinical procedure. Most commonly, researchers assume that bedside nurses will perform frequent blood draws necessary for a pharmacokinetic substudy. Unit managers, however, may not agree to commit nurses' time to this task, depending on the number and timing of the draws. During surgery, a study may require recording of events or timelines which are not usually recorded. Researchers sometimes assume that operating room personnel will be able to focus on this data collection. Hospital managers and directors are often concerned that researchers assume that unit staff will transport patients within-hospital for research procedures, which can involve repeatedly moving a patient from their home unit to Radiology or elsewhere and then back to the home unit. For a large hospital, this can involve considerable staff time spent away from the home unit, which may affect unit operations. Occasionally, an investigator requests that a hospital temporarily or permanently store blood or tissue samples. Since hospitals are not necessarily prepared to store large numbers of samples for extended periods, we address each such request on a case-by-case basis.

\section{Investigational Product}

Hospital reviewers need to consider an assortment of challenges relating to the receipt, storage, dispensing, and accountability recording of investigational products. If a hospital pharmacy will be dispensing an investigational drug, then pharmacists need to anticipate its arrival from the sponsor and know storage and other sponsor, study and FDA requirements. Pharmacists also need to know if they are expected to prepare placebo pills. If a drug is an agent that an external pharmacy needs to prepare and compound, the chain of custody documentation of the drug as it moves from manufacturer to external pharmacy to hospital pharmacy needs to be clear.

While academic hospitals usually have research-knowledgeable pharmacists, community-based hospital pharmacists may not be familiar with the special requirements of the FDA or sponsor, such as securing investigational products separately from marketed products, recording batch numbers, maintaining accountability logs, and following procedures for return or destruction of remaining product upon study closure. In our hospital system, 2 Research Pharmacists in the Investigational Drug Pharmacy at the academic teaching hospital serve as expert advisers to pharmacists of the community hospitals.

If a research study involves use of an investigational device, hospital reviewers need to consider what the device is, how it should be secured if stored on the unit, and how to document storage and accountability.

Biologics and radioactive materials can present unique challenges. For instance, our system requires researchers to provide hospitals with chain of custody documentation, similar to that used for organ transplants, when patient biologicals leave the hospital (for instance, for processing at a nearby accredited Cell Processing Facility) and returned to the hospital for infusion back into the patient, to confirm that the right product was returned to the hospital and infused into the right patient.

\section{Unaffiliated Principal Investigators and Other Personnel}

Increasingly, researchers who are not affiliated with our hospital system have inquired about conducting studies in 1 or more of our hospitals. We have been quite surprised by the number of inquiries from researchers or sponsors who presume we will grant immediate permission for them to access our patient lists for recruitment purposes, allow unidentified research team members to enter our hospitals, approve team members to conduct active recruitment of our hospital patients, and grant team members access to patient data.

Additionally, as clinical and translational research projects become increasingly multidisciplinary and involve cross-organizational collaborations, many research teams include unaffiliated personnel from other organizations, such as faculty at local or distant universities, employees of a site management organization, and employees of the city or county health department.

We do not permit clinicians who are not clinically credentialed at our hospitals to engage in interventional research within our hospital system. For studies that include any intervention that qualifies as a clinical procedure, the Principal Investigator must become clinically credentialed by the hospital or an already credentialed clinician must become the local Principal Investigator for the study, and all team members who perform clinical procedures must also become clinically credentialed.

An unusual situation occurred when researchers from a university not formally affiliated with our hospital system sought to transfer a study to 1 of our hospitals following Hurricane Ike. For 15 years, the research team had performed needle muscle biopsies, for which they had received training and credentialing at their home institution. Our hospital's Chief Medical Officer, however, did not feel comfortable accepting the credentialing performed at another institution and required the external researchers to apply for privileges through our hospital's credentialing process. The original credentialing documentation at their home institution was unavailable, in any case, due to flooding and building closures, so the researchers worked through the night to complete our applications. The hospital credentialing committee came to order on short notice and completed the credentialing process in record time, allowing the research

2010 Society of Hospital Medicine DOI 10.1002/jhm.694 Published online in wiley InterScience (www.interscience.wiley.com). 
team to see the study participants at our hospital with only a few missed visits and few study deviations for the Principal Investigator.

For research team members who will be performing no clinical procedures, our hospital system has a research credentialing process, discussed below.

\section{Financial Issues}

\section{Study Finances}

Researchers are typically aware of charges for the procedures that their studies most frequently require, such as labs, radiology, and research pharmacy, although they often do not inquire about the hospital's current charges, so their information may be somewhat dated when they negotiate with sponsors or submit grant applications.

When researchers plan on utilizing hospital staff to perform research-required tasks, such as blood draws or patient transport for tests or procedures, however, they rarely include financial support to the hospital in their study budgets, leaving these tasks unfunded, which can be a problem for hospital reviewers who must approve the research plan.

In our review and approval process, we seek clarification as to payment for research products. Sponsors are not permitted by law to seek payment for investigational drugs, but when a research protocol uses a standard-of-care drug for strictly research-related purposes, determining whether the sponsor, hospital, or study participant incurs financial responsibility becomes an issue requiring careful consideration. We ensure that consent forms explicitly state any special charges to the study participant. An investigational device that falls into an FDA classification that permits charges can be especially problematic. Often the devices are expensive; the hospital must purchase them in batches and pay immediately. If the researcher does not use all the devices, the unused ones sometimes cannot be returned. If investigational devices are more costly than standard-ofcare devices, then the hospital could incur substantial losses in billings, since hospital charges are diagnosis and procedure code dependent and usually not adjusted for device cost.

Our standardized financial assessment has led to more beneficial arrangements with sponsors for the return and reimbursement of unused products and more informed hospital decisions as to whether to conduct a specific medical device clinical trial.

We also carefully review consent form language to clarify who incurs costs for research-related adverse events and research injuries. For investigational products with extensive potential side effects or studies enrolling very ill patients, the costs associated with adverse events can be extremely high.

Investigators are affiliated with our hospitals, not employed by them, so our hospital system does not have budget agreements with funding organizations, but relies on these external entities to fund the studies adequately. Some investigator-initiated studies may be funded by the investigator's organization, such as a private practice or university, but in our experience such funds are sometimes depleted before study completion.

\section{Hospital Financial Information as Study Data}

As a part of the study protocol, researchers occasionally request patient-related cost data pertinent to specific procedures or to the treatment of certain medical conditions, hospital charges and payments received, or other financial data. We forward these research requests to a hospital Finance Chief to determine the appropriateness of releasing the requested data and, if approved, how the hospital will extract and present the data sets to the investigator.

\section{Hospital Risk Issues \\ Research Equipment}

Our academic teaching hospital and the university medical school affiliated with it are distinct organizations, so a particular risk issue arises for the hospital when a researcher wishes to transport and use nonhospital equipment on the hospital premises. Our hospital system has responsibility to ensure appropriate and safe operation of equipment. Consequently, our hospital system review identifies any proposed use of externally-owned equipment and involves the system and hospital Risk Officers in assessing such use for risk to patients. Specifics addressed include ownership of equipment, whether the research team or hospital staff will operate it; whether the operators have received or will receive training, and the potential risks to patients of equipment malfunctions. Upon determination that individuals will operate the equipment appropriately with minimal risks to study participants, the hospital's Biomedical Department performs their standard safety check prior to the equipment's use in the hospital.

\section{Clinical Data Generated by NonHospital Equipment}

Bringing external equipment into a hospital has a rarely anticipated consequence: the generation and storage of patient data. Our hospital system review determines whether these data constitute source data per FDA and ICH GCP definitions, whether the data are clinically pertinent, and whether the data need to go into the patient's hospital medical record. For example, if a university faculty researcher brings a vital signs monitor into the hospital to collect and electronically record data from hospital patients enrolled in a study, we may require that the research team print the data for insertion into the patient's hospital medical record.

\section{Surveys of Hospital Employees}

A research study that seeks to survey or interview hospital system employees raises a different type of institutional concern. In such a case, system and hospital Human 
Resources Department (HR) personnel review the planned study, paying close attention to how the research team will recruit employees and what type of information the team will request from them. HR does not want employees feeling that they must participate in a study simply because it takes place in their facility and wants to protect the identity of employees participating in anonymous surveys. On occasion, Institute staff distribute surveys (and sometimes collect them when completed), providing an identity firewall between the employee who elects to participate and the researcher.

HR may also limit researchers from asking especially personal questions or questions inquiring about the recruitment, hiring, and retention practices of their employer. HR must consider whether the study design and survey questions raise liability concerns. Studies that include employee focus groups or one-on-one interviews raise issues pertaining to the purpose and content of the focus groups or interviews, mechanisms to address special issues or complaints that may arise during them, and determination of whether the employees may participate during paid time as opposed to participating while "off-the-clock."

\section{Nonclinical Research Credentialing Process}

It is vital that hospitals know who will be on hospital premises for a research study and what activities they will be engaging in. If a research team member is not already affiliated with our hospital system and will be engaging in noninterventional research activities, such as conducting the research consent process, administering a survey, or providing educational materials, our hospital system review initiates a nonclinical research credentialing process. The Institute's Manager of Clinical Research Operations assesses the team members' qualifications, reviews their resumes, interviews each of them, and discusses exactly what activities they will be engaging in and their training and experience. In addition to evaluating their qualifications, the hospital must determine how such individuals will be identified once inside the hospital, including what type of badge (employee, contractor, or visitor) the hospital will provide them. Upon successful completion of the research credentialing process, the Institute explicitly names the approved individuals in the Letter of Approval to the Principal Investigator, with copies to the affected hospitals' units.

\section{Discussion}

Our hospital research review and approval process is critical to ensuring that only safe and regulatory-compliant research activities occur within our hospital system, but the review and approval process, with its many steps and numerous reviewers, can be cumbersome. There is no substitute for human beings reading and understanding the protocol, consent forms for patient involvement in a study, the proposed use of protected health information, Investigator's Brochure, Research Application Form, and other study documents and then identifying pertinent issues and resolving them, and this process does require significant staff time.

We have improved (continuously) the Research Application Form to help in the crucial initial gathering of information about studies' operational needs. We have also converted from a predominantly paper-based review process to the widespread use of electronic documents, but we have not automated the distribution process for these electronic documents and a staff person must still do this through email.

Despite our efforts to improve the review process, investigators are sometimes frustrated with it, particularly if someone identifies a new issue late in the process, or if the hospital system's approval for the study lags behind the IRB approval by more than a few days.

Currently, the hospital system provides the majority of study approvals to the Principal Investigator within 2 weeks of IRB approval, with some approvals provided within 1 day of IRB approval and others as long as 3 months afterward. Delays in hospital approval can be due to a study lacking required approval from a Department of Defense IRB, the FDA not providing permission to proceed with a study, the absence of an executed contract with a vendor to pick up and dispose of radioactive waste from the investigational product and many other factors. Of course, when the research team can respond in timely fashion to inquiries or issues that we have raised, that assists all of us in completing the review and approval process as quickly as possible.

The review and approval process benefits hospital patients, hospital personnel who will be supporting studies, and hospitals as institutions. Thinking through, planning, and preparing for study operations, particularly for studies taking place in an ICU, benefits the research team, hospital personnel, and the patients. Overall, the hospital system's research review and approval process affords many protections to our patients and reduces risks to the hospital system while permitting research studies to be conducted within its varied healthcare facilities.

We encourage researchers not to view today's modern hospital as "bricks and mortar," but as an institution with deep responsibility for safety on hospital premises. Hospitals must meet over a thousand Joint Commission standards, set goals for patient outcomes, measure and report on quality indicators, protect patient confidentiality, maintain the safety an ever-expanding array of simple and complex equipment, maintain, check and document contents of adult and pediatric crash carts, have 24-7 code teams atthe-ready, create, maintain and store patient medical records securely, transport patients, and much more.

\section{Conclusion}

Our hospital system, in accordance with a system-wide policy, engages in a comprehensive review and approval process for any human participant research that has been proposed to be conducted within one or more of our facilities. The process focuses primarily on patient safety within the

2010 Society of Hospital Medicine DOI 10.1002/jhm.694 Published online in wiley InterScience (www.interscience.wiley.com). 
hospital premises, operational study issues, financial issues and hospital risk issues. This process decreases risks to the patients, researchers, and hospital facilities engaging in human participant research.

\section{Address for correspondence and reprint requests:}

Cheryl M. Chanaud, PhD, CCRP, 6411 Fannin St., Houston, TX 77030; Telephone: 713-704-5647; Fax: 713-704-5124; E-mail: cheryl.chanaud@memorialhermann.org Received 12 October 2009; revision received 18 January 2010; accepted 14 February 2010.

\section{References}

1. Code of Federal Regulations Part 45, Title 46.

2. Code of Federal Regulations Part 50, Title 21.

3. Flanders SA, Kaufman SR, Nallamothu BK, Saint S. The University of Michigan Specialist-Hospitalist Allied Research Program: jumpstarting hospital medicine research. J Hosp Med. 2008;3:308-313.

4. Moore C, Goldberg S. Successfully developing a cardiovascular research program in a community hospital. J Cardiovasc Manag. 2004;15:13-19.

5. Jamerson PA. Developing an infrastructure for research in a free-standing hospital. J Nurs Adm. 2007;37:295-301.
6. Schneider KM, O’Donnell BE, Dean D. Prevalence of multiple chronic conditions in the United States' Medicare population. Health Qual Life Outcomes. 2009;7:82.

7. Getz KA, Wenger J, Campo RA, Seguine ES, Kaitin KI. Assessing the impact of protocol design changes on clinical trial performance. Am J Ther. 2008;15:450-457.

8. Roche K, Paul N, Smuch B, et al. Factors affecting workload of cancer clinical trials: results of a multicenter study of the National Cancer Institute of Canada Clinical Trials Group. J Clin Oncol. 2002;20: 545-556.

9. Craft BS, Kurzrock R, Lei X, et al. The changing face of Phase 1 Cancer Clinical Trials: new challenges in study requirements. Cancer. 2009; 115(8):1592-1597.

10. U.S. Census Bureau. Annual Estimates of the Resident Population for Counties of Texas: April 1, 2000 to July 1, 2008. Available at: http:// www.census.gov/popest/counties/tables/CO-EST2008-01-48.xls. Accessed February 2010

11. Harris County Texas. Available at: http://www.gis.hctx.net. Accessed February 2010 .

12. Accreditation Program: Hospital. Available by purchase from The Joint Commission on Accreditation of Healthcare Organizations. 2008.

13. International Conference on Harmonisation. Available at: www.ich.org/ cache/compo/276-254-1.html. Accessed February 2010. 